\title{
Traitement lexicographique bilingue des mots composés du lexique culinaire grec moderne
}

Modern Greek Culinary Lexis Compounds and their Treatment in Bilingual Lexicography

Georges Galanès

\section{OpenEdition}

\section{Journals}

Édition électronique

URL : https://journals.openedition.org/ceb/7040

DOI : $10.4000 /$ ceb.7040

ISSN : 2261-4184

Éditeur

INALCO

Édition imprimée

ISBN : 978-2-85831-230-6

ISSN : 0290-7402

\section{Référence électronique}

Georges Galanès, «Traitement lexicographique bilingue des mots composés du lexique culinaire grec moderne », Cahiers balkaniques [En ligne], Hors-série | 2016, mis en ligne le 14 mars 2017, consulté le 06 juillet 2021. URL : http://journals.openedition.org/ceb/7040 ; DOI : https://doi.org/10.4000/ceb. 7040

Ce document a été généré automatiquement le 6 juillet 2021.

\section{cc) (1) 8}

Cahiers balkaniques est mis à disposition selon les termes de la Licence Creative Commons Attribution - Pas d'Utilisation Commerciale 4.0 International. 


\title{
Traitement lexicographique bilingue des mots composés du lexique culinaire grec moderne
}

\author{
Modern Greek Culinary Lexis Compounds and their Treatment in Bilingual \\ Lexicography
}

Georges Galanès

1 Cette communication portera sur les mots composés du lexique culinaire grec moderne et leur traitement en lexicographie bilingue (français-grec). Il s'agira de répertorier les unités lexicalisées du grec moderne (composés lexicaux unifiés) et de voir dans quelle mesure le traitement lexicographique les associe aux diverses locutions du français. Il sera notamment question de la présence de ce type d'unités lexicalisées du grec moderne dans un dictionnaire français-grec, où les locutions correspondantes ne sont pas lexicalisées, ce qui n'est pas le cas dans des dictionnaires thématiques spécialisés (par exemple, "beurre blanc », "beurre de cacao », "beurre composé », "beurre de Gascogne » constituent des entrées à part dans le Larousse gastronomique, alors qu'elles sont de simples locutions à l'intérieur de l'article beurre dans le Larousse).

2 La composition en grec est un phénomène qui relève du lexique et non pas de la syntaxe, ce qui est moins vrai pour d'autres langues, y compris le français. J'ai utilisé le dictionnaire du grec moderne de l'Université de Thessalonique (édition de 1999 mise à jour), parce qu'il intègre dans la nomenclature des composants (qu'ils soient à gauche ou à droite), alors que celui du Centre de lexicologie ne le fait que de façon très occasionnelle et quasiment pas du tout pour le lexique culinaire. Pour la correspondance en français j'ai utilisé le Dictionnaire français-grec moderne Larousse édité par les éditions Patakis en 2012. Les deux dictionnaires sont de taille plus ou moins égale.

3 J'ai exclu de mon corpus a) les verbes, peu intéressants pour mon propos, qu'ils soient formés à partir d'un nom composé avec un suffixe verbal ( $\alpha \lambda \alpha \tau o \pi \imath \pi \varepsilon \rho \omega ́ v \omega$ [saler et poivrer]) ou à partir d'une tête verbale ( $\alpha v \gamma o \kappa o ́ \beta \omega$ [battre un œuf]) et b) les adjectifs, 
relativement peu nombreux et souvent correspondant à des composés français

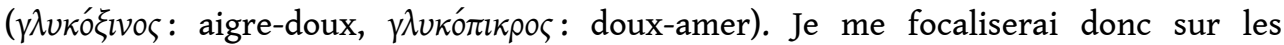
substantifs.

Il y a deux grandes classes de substantifs composés en grec moderne, que l'on retrouve dans le lexique culinaire : a) les coordinatifs (composés polycentriques de type dvandva) et $b$ ) les subordinatifs (composés monocentriques le plus souvent déterminatifs de type tatpurusha).

Les coordinatifs à deux composants font généralement précéder l'élément qui constitue la base de l'ensemble ou qui est censé être plus important ou en plus grande quantité

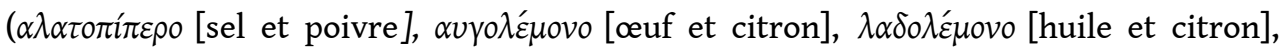

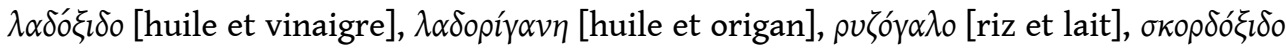
[ail et vinaigre], $\psi \omega \mu$ uópl [pain et fromage] pour les noms lexicalisés et

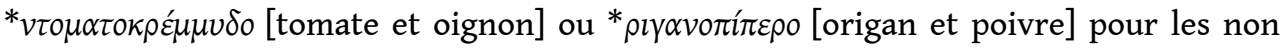
lexicalisés). La classification des composés de type $\pi \rho \alpha \sigma o ́ p v \zeta o$ [poireaux et/au riz] ou $\sigma \pi \alpha v \alpha \kappa o ́ p v \zeta o$ [épinards et/au riz] (tous les deux lexicalisés), semble plus délicate. S'agitil d'un riz aux poireaux ou aux épinards ou bien d'un mélange des deux ingrédients où, sur le modèle des autres composés de ce type le premier élément serait aussi l'élément de base ? Dans le premier cas, on aurait un subordinatif avec une tête à droite, alors que dans le second il s'agirait d'un coordinatif avec un accent à gauche et c'est ce qui me semble le plus probable. L'élément - $\rho v \zeta_{0}$ [riz] est par ailleurs très productif, puisque j'ai

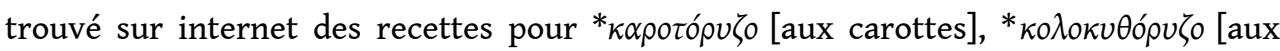
courgettes], * ${ } \rho \varepsilon \mu \mu v \delta o ́ \rho v \zeta o$ [aux oignons], ${ }^{*} \lambda \alpha \chi \alpha v o ́ \rho v \zeta_{0}$ [au chou], * $\mu \varepsilon \lambda u \tau \zeta \alpha v o ́ \rho v \zeta_{0}$ [aux

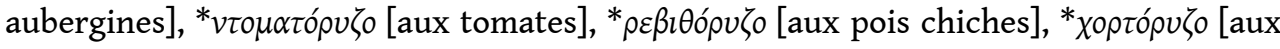
herbes] et même un curieux * $\pi \alpha \tau \alpha \tau o ́ p v \zeta o$ [aux pommes de terre]. Cet élément - $\rho v \zeta o$ est absent du dictionnaire grec et, parmi les éléments à gauche, seuls $\lambda \alpha \chi \alpha v o-$ (associé dans

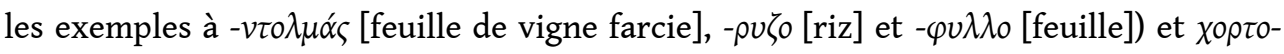

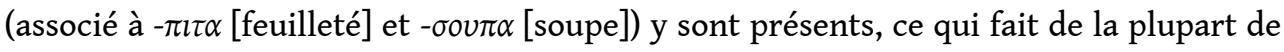
ces mots plus que des absents, des éléments impossibles à recomposer.

6 Mais tous les coordinatifs à deux composants ne font pas précéder l'élément qui constitue la base de l'ensemble ou qui est censé être plus important ou en plus grande

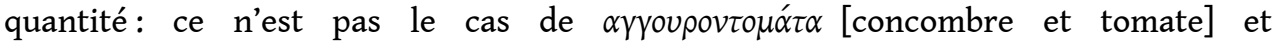

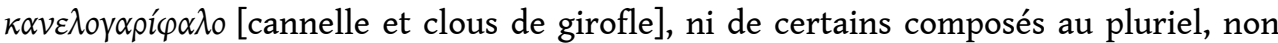

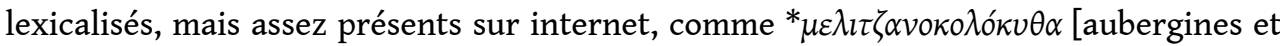
courgettes] ou ${ }^{*} \pi \alpha \tau \alpha \tau o \kappa o \lambda o ́ \kappa v \theta \alpha$ [pommes de terre et courgettes]. Par ailleurs, il semble

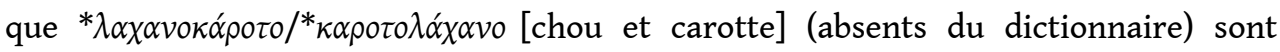
interchangeables avec une petite remarque : l'accentuation du second atténue l'unité des deux composants. Le cas de * $\kappa_{0} \rho \delta o \kappa \rho \varepsilon ́ \mu \mu v \delta o$ [ail-oignon] (absent du dictionnaire grec) est intéressant, dans la mesure où le mot peut avoir le sens d'un assortiment d'oignon et d'ail (la base semble être le second élément), mais il signifie aussi l'échalote et, dans ce cas, il s'agit d'un composé subordinatif.

7 Les mots lexicalisés que je viens de mentionner ont peu de chances d'apparaître dans un dictionnaire bilingue français-grec (c'est le cas du dictionnaire Larousse). Ils correspondent à des locutions nominales de type « sel et poivre » ou « sauce à l'œuf et au citron ", avec lesquelles nous sommes familiers, mais qui sont absentes évidemment des listes d'exemples des dictionnaires français, absence qui est répercutée dans les dictionnaires bilingues. Seul $\rho v \zeta o ́ \gamma \alpha \lambda$ trouve sa place comme traduction de « riz au 
lait » (article : riz) et dans la locution $\gamma \lambda v \kappa o ́ ~ \rho v \zeta o ́ \gamma \alpha \lambda o ~ \mu \varepsilon ~ \alpha v \gamma \alpha ́$ [riz au lait sucré aux œufs] (on aurait pu traduire aussi par $\gamma \lambda v \kappa i \alpha ́ \alpha ~ \rho v \zeta o ́ \pi \imath \tau \alpha$ [flan au riz], avec une marque d'approximation) proposée comme traduction de "gâteau de riz » (article : gâteau). La question qui se pose est de savoir dans quelle mesure un dictionnaire bilingue, en tant que lieu de rencontre de deux cultures, devrait prendre en compte la langue cible dans la conception de la structure des articles.

Cette question est particulièrement pertinente concernant les composés subordinatifs, lexicalisés ou non lexicalisés (cette classe est extrêmement productive en grec). Dans les subordinatifs, les composants sont réunis sur la base de la subordination sémantique. Il s'établit entre eux des relations de soumission d'un composant envers l'autre. Cela tient à la présence d'une tête sémantique unique. Il s'agit en grec de composés endocentriques déterminatifs, le plus souvent formant un couple déterminant-déterminé, avec l'élément déterminé à droite.

Parmi ces composés subordinatifs on trouve dans le dictionnaire grec des cas uniques, dont on pourrait déduire que les composés ne sont pas productifs: $\alpha v \gamma o \tau \alpha ́ p \alpha \chi o$ [poutargue], $\alpha v \gamma o ́ \varphi \varepsilon \tau \alpha$ [pain perdu], $\gamma \alpha \lambda \alpha \kappa \tau o \mu \pi о v ́ \rho \varepsilon \kappa o$ [feuilleté à la crème], $\lambda \alpha \delta o \tau u ́ \rho t$

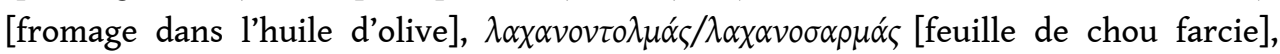

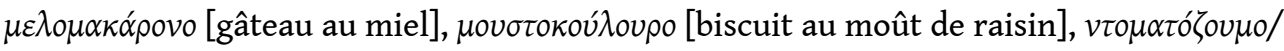

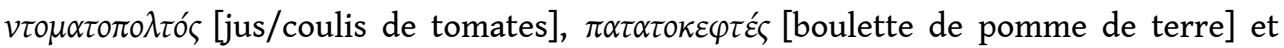

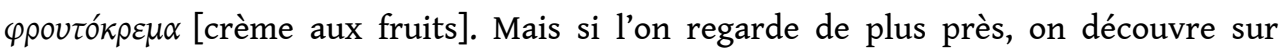

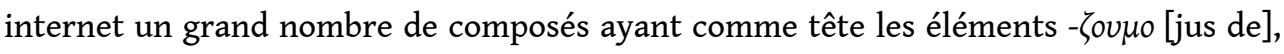

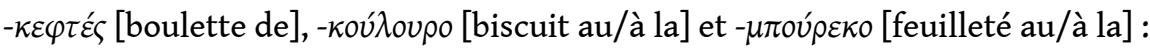

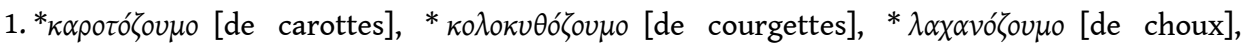

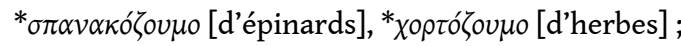

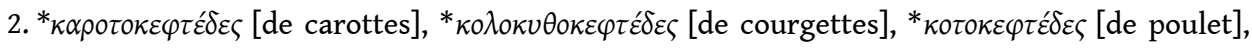
${ }^{*} \kappa \rho \varepsilon \mu \mu v \delta о \kappa \varepsilon \varphi \tau \varepsilon \delta \varepsilon \varsigma$ [d'oignons], ${ }^{*} \lambda \alpha \chi \alpha v o \kappa \varepsilon \varphi \tau \varepsilon \delta \varepsilon \varepsilon \zeta$ [de chou], * $\mu \alpha v i \tau \alpha \rho \circ \kappa \varepsilon \varphi \tau \varepsilon \delta \varepsilon \zeta$ [de champignons], ${ }^{*} \mu \alpha \rho o v \lambda o \kappa \varepsilon \varphi \tau \varepsilon \dot{\varepsilon} \varepsilon \varsigma$ [de laitue], ${ }^{*} \mu \varepsilon \lambda \lambda_{i} \zeta \alpha v o \kappa \varepsilon \varphi \tau \varepsilon \delta \varepsilon \varsigma$ [d'aubergines],

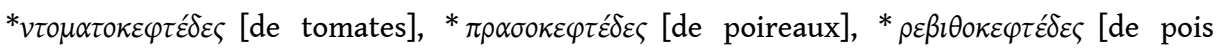

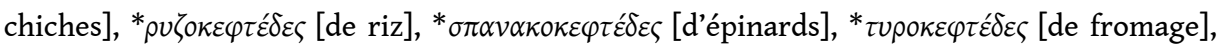

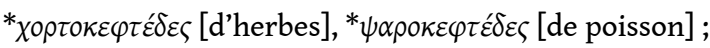

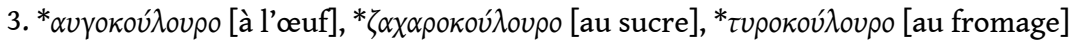

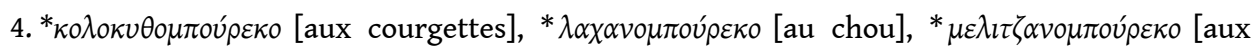

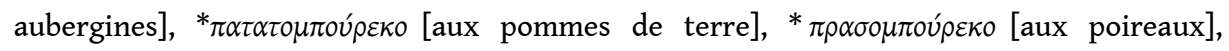

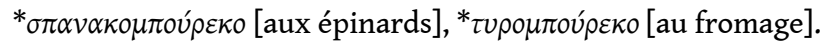

10 Or, ni ces mots ni les éléments qui les composent (sauf les déterminants $\zeta \alpha \chi \alpha \rho 0^{-}$, $\kappa o \tau 0^{-}$ $\lambda \alpha \chi \alpha v 0^{-}, \chi о \rho \tau 0_{-}$et $\left.\psi \alpha \rho 0_{-}\right)$ne sont présents dans le dictionnaire grec. Des déterminés

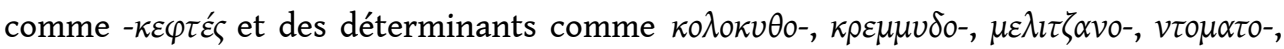

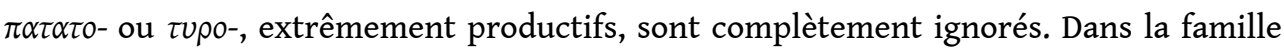
du vinaigre, $\mu \eta \lambda \hat{́}_{\xi} \delta_{0}$ [vinaigre de cidre] est absent du dictionnaire.

11 Les autres composés subordinatifs présents dans le dictionnaire grec peuvent être

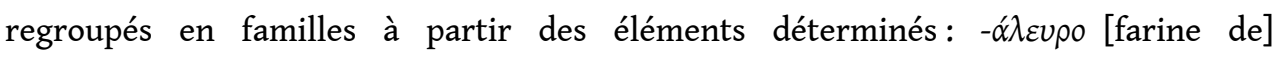

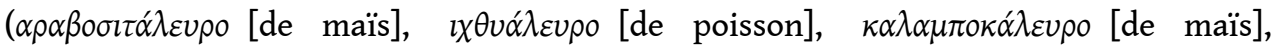

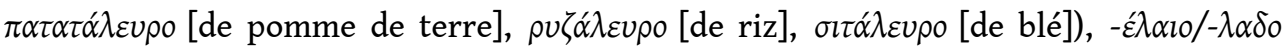

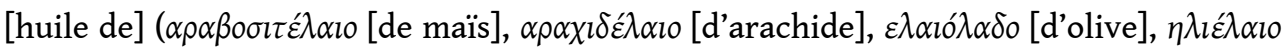

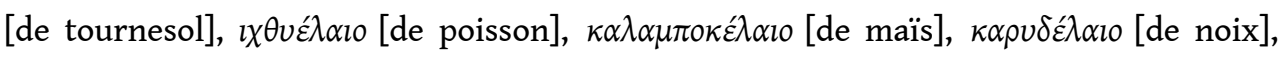

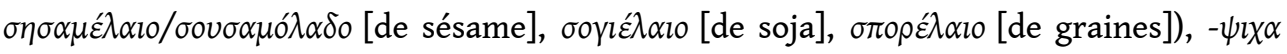


[pulpe de] ( $\alpha \mu v \gamma \delta \alpha \lambda o ́ \psi \imath \chi \alpha$ [d'amande], $\kappa \alpha \rho v \delta o ́ \psi \imath \chi \alpha$ [de noix]), $-\psi \omega \mu$ [pain de ou au/à la]

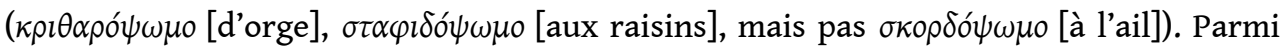

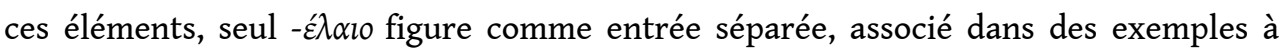

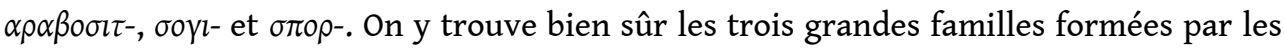
déterminés - -iı [feuilleté au/à la, parfois "gâteau ou tarte au/à la "], - $\sigma \alpha \lambda \alpha \dot{\tau} \alpha \alpha$ [salade

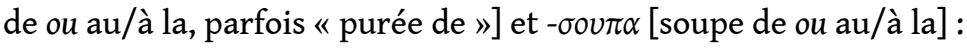

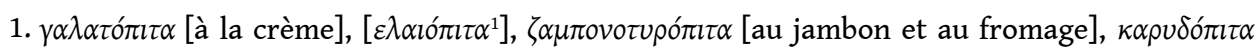

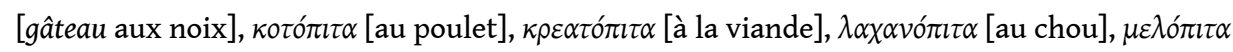

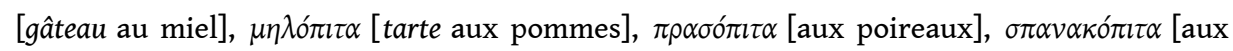

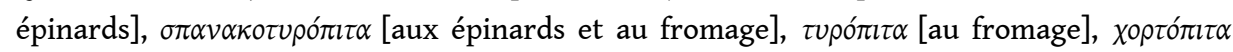
[aux herbes] ;

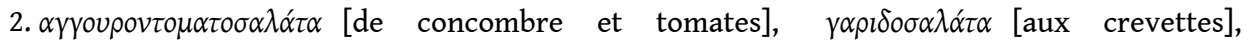

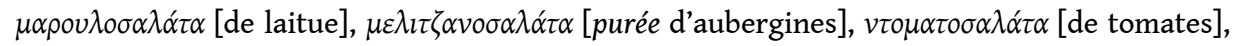

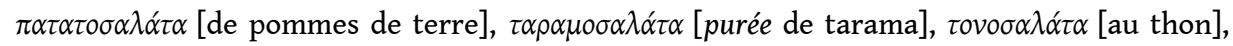
$\varphi \rho \circ v \tau o \sigma \alpha \lambda \alpha \dot{\tau} \alpha \alpha$ [de fruits];

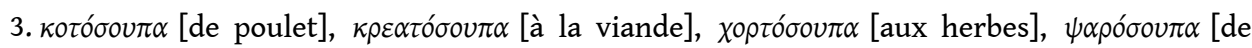
poisson]).

Mais là encore, pour ces trois grandes familles de composés, on peut trouver sur internet une quantité impressionnante de déterminants :

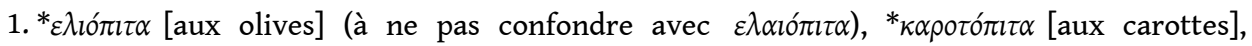
${ }^{*} \kappa \circ \lambda$

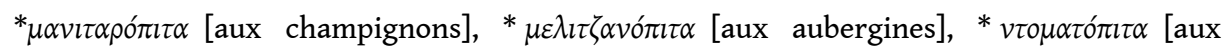
tomates], ${ }^{*} \pi \alpha \tau \alpha \tau o ́ m \imath \tau \alpha$ [aux pommes de terre];

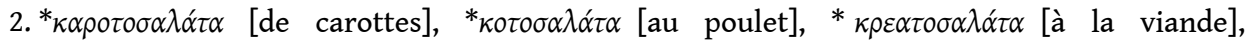

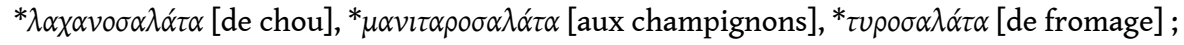

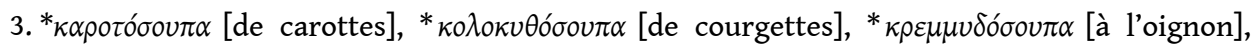

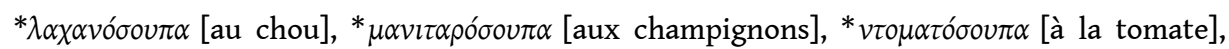

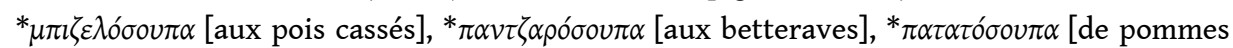
de terre], ${ }^{*} \pi \rho \alpha \sigma o ́ \sigma o u \pi \alpha$ [de poireaux], ${ }^{*} \rho \varepsilon \beta \imath$ $\beta_{0} \sigma \sigma o v \pi \alpha$ [aux pois chiches].

Il est vrai que ces trois familles sont présentes à travers des articles dédiés à leur constituant déterminé dans le dictionnaire grec :

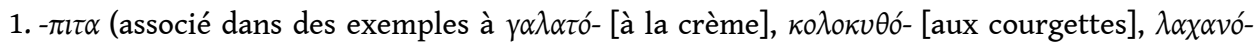

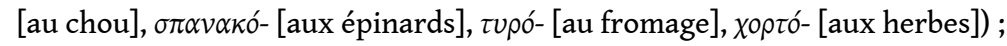

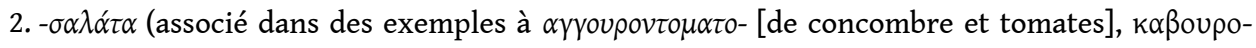
[de crabe], $\mu \alpha \rho o u \lambda_{o-}$ [de laitue], $v \tau o \mu \alpha \tau o-$ [de tomates], $\pi \alpha \tau \alpha \tau o-$ [de pommes de terre], $\tau \alpha \rho \alpha \mu o-$ [de tarama], זupo- [de fromage]);

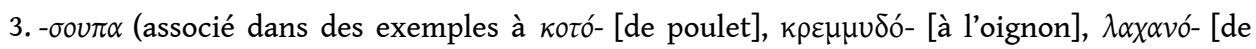

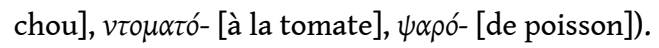

En fait, ces exemples reprennent pour la plupart les déterminants des composés présents dans la nomenclature du dictionnaire, seuls les éléments en italiques $\kappa \alpha \beta o v \rho o-$ et $\tau v \rho o-$ apportant un constituant nouveau à $-\sigma \alpha \lambda \alpha \dot{\tau} \tau \alpha$, seuls les éléments $\kappa \rho \varepsilon \mu \mu v \delta o ́-$,

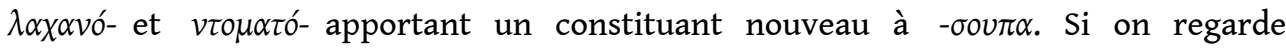
maintenant du côté des déterminants constituant des articles à part dans le dictionnaire, on ne trouve que $\lambda \alpha \chi \alpha v o$ - (ci-dessus en gras), associé dans des exemples à

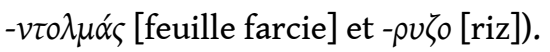


Pour résumer, sont présents comme entrées séparées dans le dictionnaire grec les

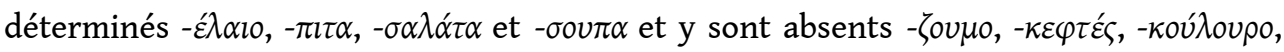
$-\mu \pi о u ́ \rho \varepsilon \kappa o ~ e t ~-\psi \omega \mu$. En ce qui concerne les déterminants, on y trouve $\alpha \lambda \alpha \tau o-[s e l]$,

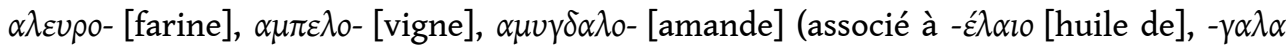
[lait de], $-\lambda \alpha \delta o$ [huile de], $-\psi \imath \chi \alpha$ [pulpe de], - $\pi \imath \tau \alpha$ [gâteau de]), $\gamma \alpha \lambda \alpha \kappa \tau$ - [crème] (associé à

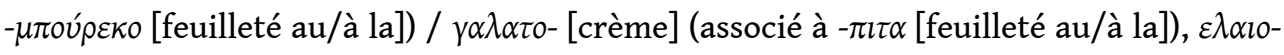

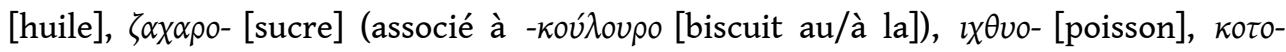

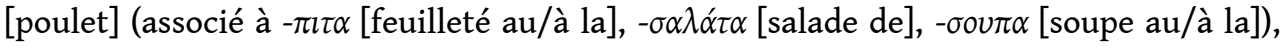

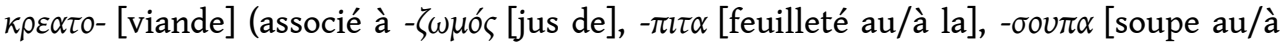

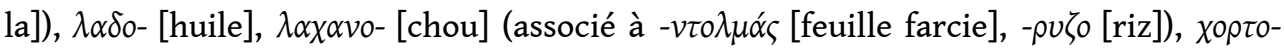

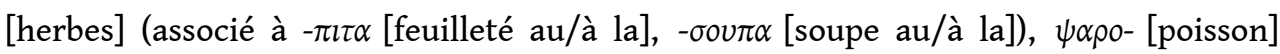
(associé à $-\lambda \alpha \delta o$ [huile de], - $\sigma 0 v \pi \alpha$ [soupe de]) et $\psi \omega \mu$ o- [pain], mais on n'y trouve pas

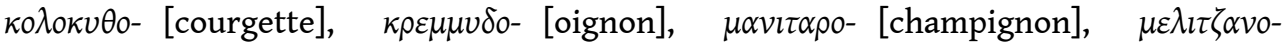

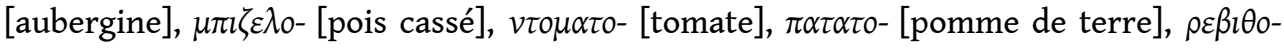
[pois chiche] et $\tau$ voo- [fromage].

On constate une grande divergence entre les déterminés du grec et ceux du français (présents dans le dictionnaire bilingue au moyen de locutions nominales). Sur l'ensemble du dictionnaire français-grec Larousse-Patakis, seulement 29 composés grecs de ce type apparaissent dans la traduction d'exemples de locutions nominales avec préposition à ou de, équivalent français de ce type de composés.

On peut les classer dans deux listes. Tout d'abord ceux où le déterminant indique principalement l'ingrédient à l'origine du produit (les composés grecs utilisés dans la traduction sont en gras - quand la traduction proposée ne contient pas de mot composé je l'ai omise) :

\begin{tabular}{|c|c|}
\hline beurre : & $\sim$ d'anchois, $\sim$ d'arachide/de cacahuètes $\varphi v \sigma \tau \imath \kappa o ß o v ́ \tau v \rho o, ~ \sim$ de cacao, $\sim$ d'escargot \\
\hline farine : & 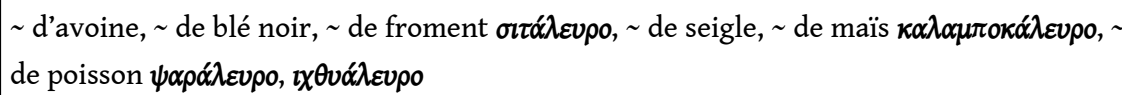 \\
\hline huile : & 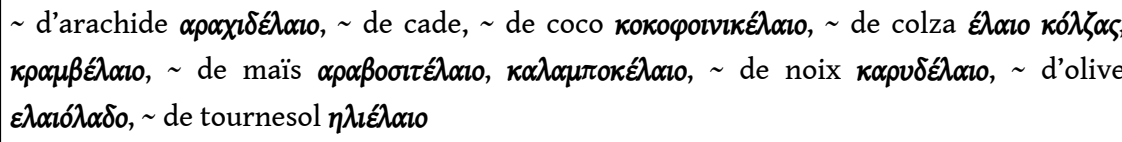 \\
\hline vinaigre : & $\sim$ de cidre $\mu \eta \lambda \hat{\xi} \zeta \delta$, à l'estragon \\
\hline
\end{tabular}

Ensuite ceux où le déterminant indique l'ingrédient principal contenu en grande quantité dans le produit (les composés grecs utilisés dans la traduction sont en gras) :

\begin{tabular}{|c|c|}
\hline chausson: & 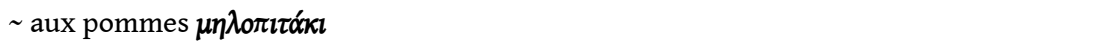 \\
\hline feuilleté : & $\sim$ aux asperges $\sigma \pi \alpha \rho \alpha \gamma \gamma o ́ \pi \imath \tau \alpha$ \\
\hline galette: & 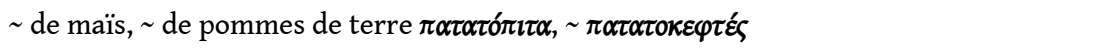 \\
\hline gâteau : & $\sim$ de semoule, $\sim$ de riz $\gamma \lambda v \kappa o ́ \rho v \zeta o ́ \gamma \alpha \lambda o \mu \varepsilon \alpha v \gamma \alpha \alpha, \sim$ aux noix $\kappa \alpha \rho v \delta o ́ \pi \imath \tau \alpha$ \\
\hline pain : & 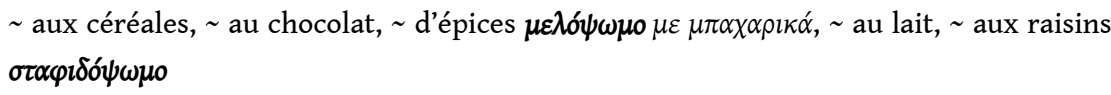 \\
\hline pâte : & $\sim$ d'amandes $\alpha \mu v \gamma \delta \alpha \lambda \operatorname{có}^{\prime} \alpha \sigma \tau \alpha, \sim$ d'anchois, $\sim$ de coing $\kappa v \delta \omega v o ́ \pi \alpha \sigma \tau \tau, ~ \sim$ de fruits \\
\hline
\end{tabular}




\begin{tabular}{|c|c|}
\hline riz : & $\sim$ au lait $\rho v \zeta o ́ \gamma \alpha \lambda$ \\
\hline salade : & $\sim$ de fruits $\varphi \rho o v \tau o \sigma \alpha \lambda \alpha ́ \tau \alpha$ \\
\hline soupe : & 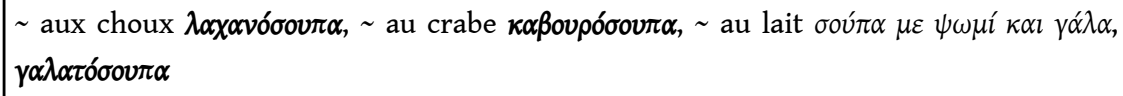 \\
\hline tarte : & 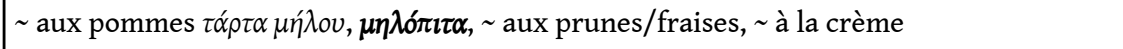 \\
\hline tourte : & 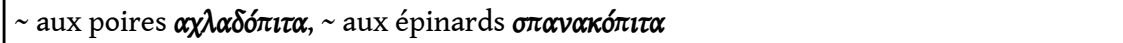 \\
\hline velouté : & 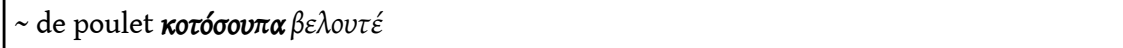 \\
\hline
\end{tabular}

19 Si dans la traduction de ces exemples on trouve des mots composés du grec moderne, il s'agit souvent de tentatives d'appropriation de recettes qui divergent parfois considérablement. Si l'on voulait associer les trois grandes familles de déterminés des composés grecs à des locutions françaises, on découvrirait, tout d'abord, que - $\pi \imath \tau \alpha$ doit être remplacé par chausson, feuilleté, galette, gâteau, tarte et tourte, mais que dans les exemples de locutions fournies par Larousse la réalité culinaire grecque est absente et très éloignée de celle représentée dans le dictionnaire bilingue. Pour les deux autres familles, si productives, mais tellement peu présentes, même dans un dictionnaire grec qui intègre dans sa nomenclature des constituants de mots composés (déterminants et déterminés), on constate que le dictionnaire bilingue donne un minimum d'exempleslocutions, parfois très loin là aussi de la réalité culinaire grecque. Pour compléter l'inventaire des locutions nominales de type déterminé/déterminant du français, et se faire une meilleure idée si besoin était de l'écart entre les deux cuisines, voici les déterminés du français où on ne trouve pas de trace de mot composé grec:

\begin{tabular}{|c|c|}
\hline compote : & $\sim$ (de fruits), $\sim$ de pommes \\
\hline confiture : & $\sim$ de fraises/mûres, $\sim$ d'oranges \\
\hline crème : & $\sim$ au chocolat/citron, $\sim$ au beurre, $\sim(\mathrm{au})$ caramel \\
\hline crêpe : & $\sim$ au beurre/sucre, $\sim$ au jambon et aux champignons \\
\hline éclair : & $\sim$ au chocolat/au café \\
\hline flan : & à la vanille \\
\hline gelée : & $\sim$ de groseilles \\
\hline glace : & à la vanille/à l'abricot \\
\hline œuf(s) : & $\sim$ au lait \\
\hline omelette : & $\sim$ aux champignons/au fromage/au jambon, $\sim$ aux fines herbes \\
\hline pâté : & $\sim$ de canard, $\sim$ de foie \\
\hline purée : & $\sim$ de tomates/carottes, $\sim($ de pommes de terre $)$ \\
\hline sauce : & à la moutarde/aux câpres, au soja, $[\sim$ tomate $]$ \\
\hline saucisson : & à l'ail \\
\hline semoule : & $\sim$ de riz, $\sim$ de maïs, $\sim$ de blé dur \\
\hline tartine : & $\sim$ de beurre/pâté \\
\hline
\end{tabular}


Évidemment, toutes ces locutions sont traduites par des constructions appositives de

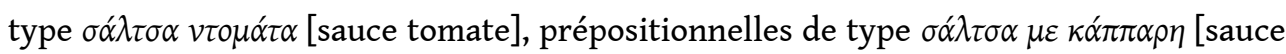

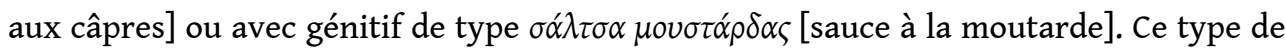
locutions existe bien en grec, en parallèle avec les mots composés, pour un grand nombre d'expressions du domaine culinaire. Mais on voit proliférer sur internet des

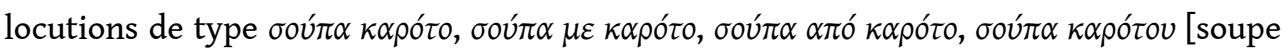
aux carottes], phénomène intéressant, mais qui est ici hors de propos et que, de toute façon, je n'aurais pas eu le temps de traiter. Il peut s'agir là d'un des effets symptomatiques du fléau du calque traductif, ou d'une volonté de marquer consciemment une différence par rapport au caractère traditionnel-populaire du composé. Il faudrait voir si des différences existent à ce niveau entre ce qui est présenté comme $\mu \alpha v i \tau \alpha \rho o ́ \pi \imath \tau \alpha, \pi \rho \alpha \sigma o ́ \pi \imath \tau \alpha, \sigma \pi \alpha v \alpha \kappa o ́ \pi \imath \tau \alpha$ d'une part et $\pi i \tau \alpha \mu \varepsilon \mu \alpha v i \tau \alpha ́ p l \alpha, \pi i \tau \alpha \mu \varepsilon$

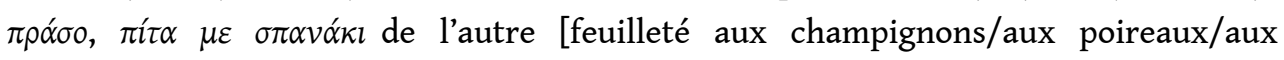
épinards].

21

Je me limiterai pour l'instant à tirer les conclusions suivantes à partir du cas des composés grecs du lexique culinaire, qui pourraient concerner plus généralement le traitement des mots composés: a) La présence des éléments composants dans les dictionnaires grecs (qu'il s'agisse de composés coordinatifs polycentriques ou de composés subordinatifs monocentriques) est d'une importance capitale, étant donné leur productivité et leur grande capacité d'intégration, ce qui n'est pas le cas actuellement; b) Il faudrait prévoir, du moins pour les plus productifs parmi eux, des équivalents en français (et dans d'autres langues bien sûr) dans les dictionnaires bilingues, dans un souci de mieux intégrer la culture de la langue cible dans le dictionnaire, mais aussi pour faire apparaitre le processus de formation de ces composés ; c) Enfin, il serait souhaitable de pouvoir mieux intégrer ce type de processus de composition dans les méthodes d'enseignement du grec moderne, puisqu'il s'agit là d'une des manifestations de la langue parmi les plus créatives et vivantes.

\section{BIBLIOGRAPHIE}

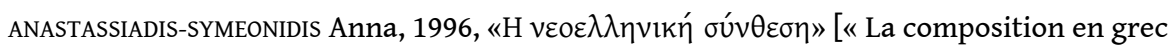

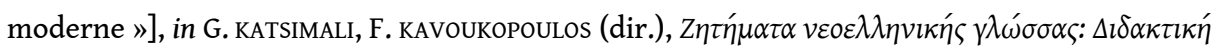
$\Pi \rho \circ \sigma^{\prime} \gamma \gamma ı \sigma \eta$ [Questions de langue grecque moderne : une approche didactique], Réthymnon : Université de Crète, 1996, p. 97-120.

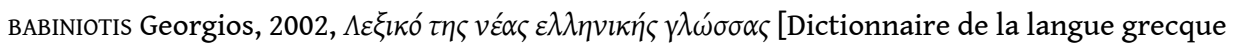
moderne], Athènes : Centre de lexicologie.

BISETTO Antonietta \& SCALISE Sergio, 2005, “The classification of compounds", Lingue e Linguaggio, IV (2), 2005, p. 319-332.

CORBIN Danielle, 1992, « Hypothèses sur les frontières de la composition nominale », Cahiers de grammaire, 17, p. 25-55.

Cahiers balkaniques, Hors-série | 2016 


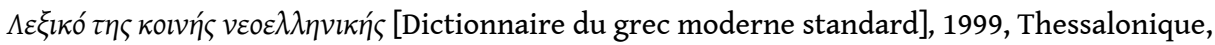
Université Aristote : Institut d'études néohelléniques, (édition revue).

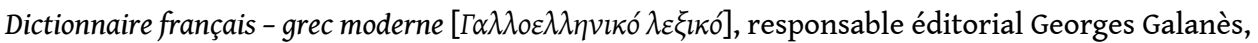
2012, Athènes : Patakis (en collaboration avec Larousse).

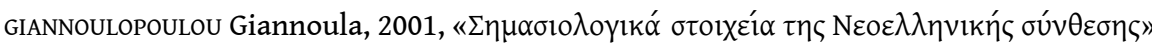

[«Éléments sémantiques de la composition en grec moderne »], in G. ANGOURAKI, A. ARVANITI, D. goutsos, J. davy, M. Karyolaimou, A. panagiotou, A. papapavlou, P. pavlou, A. roussou (dir.),

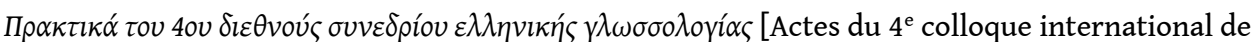
linguistique grecque], Thessalonique : University Studio Press, 2001, p. 103-111.

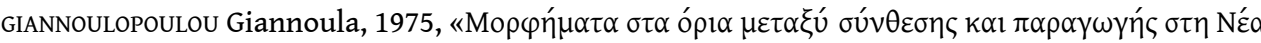

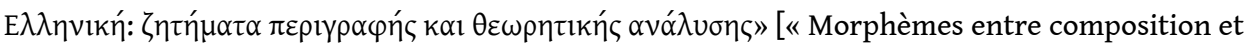
production en grec moderne : questions de description et d'analyse théorique »], in

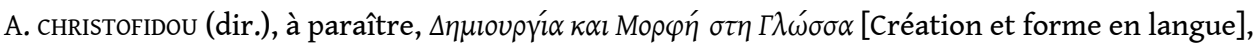

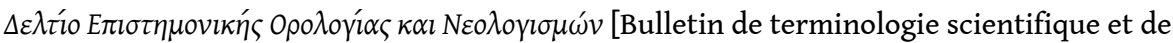
néologismes], vol. 12.

GUILBERT Louis, 1975, la Créativité lexicale, Paris : Larousse.

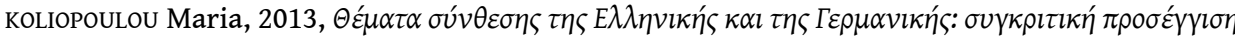
[Questions de composition en grec et en allemand : une approche comparative], thèse de doctorat, Patras.

MATHIEU-COLAS Michel, 1996, «Essai de typologie des noms composés », Cahiers de lexicologie, 69, p. 71-125.

MEL’ČUK Igor, 1997, Cours de morphologie générale, vol. IV, Montréal : P. U. Montréal.

RALLI Angela, 1992, “Compounds in Modern Greek”, Rivista di Linguistica 4 (1), pp. 143-174.

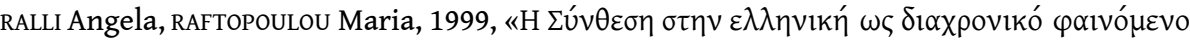

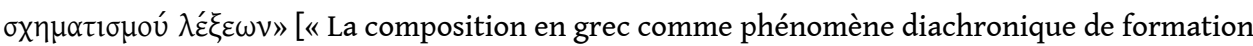

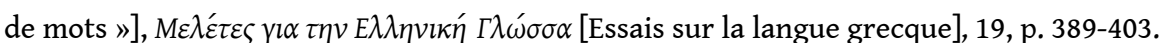

RALLI Angela, 2006, "Variation in Word Formation: The Case of the Compound Markers", in P. CUZZOLIN, M. NAPOLI (dir.), Fonologia e Tipologia Lessicale nella Storia della Lingua Greca, Milan : Franco Angeli, pp. 238-264.

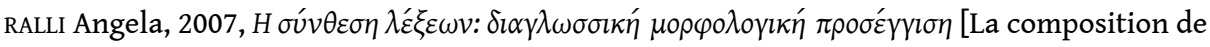
mots : une approche morphologique interlinguistique], Athènes : Patakis.

RALLI Angela, 2008, “Compound Markers and Parametric Variation", Language Typology and Universals (STUF) 61, pp. 19-38.

RALLI Angela, 2013, Greek Compounding, Dordrecht: Springer.

\section{NOTES}

1. Ce mot ne fait pas normalement partie du lexique culinaire. Il s'agit d'un terme technique pour la pâte de pulpe d'olives; néanmoins, il peut désigner parfois un feuilleté aux olives. 


\section{RÉSUMÉS}

La communication porte sur les mots composés du lexique culinaire grec moderne et leur traitement en lexicographie bilingue. Il s'agit de répertorier et classer les unités lexicalisées du grec moderne et de voir dans quelle mesure le traitement lexicographique les associe aux diverses locutions nominales du français. Dans le cadre de cette étude contrastive, il est notamment question de la présence de ce type d'unités lexicalisées du grec moderne dans un dictionnaire français-grec, où les locutions nominales correspondantes ne sont pas lexicalisées.

The paper is about Modern Greek culinary lexis compounds and their treatment in bilingual lexicography. We list and classify Modern Greek lexicalized items, then check to what extent the lexicographical treatment connects them with various French nominal locutions. Within the framework of this contrastive study, we examine especially the presence of that kind of Modern Greek lexicalized items in a French-Greek dictionary, where corresponding nominal locutions are not lexicalized.

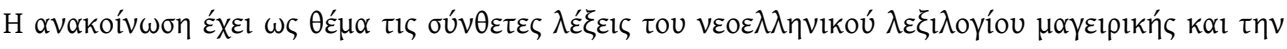

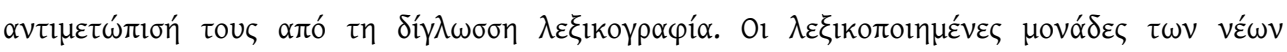

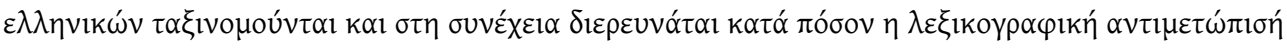

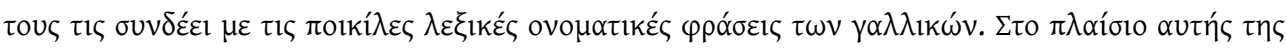

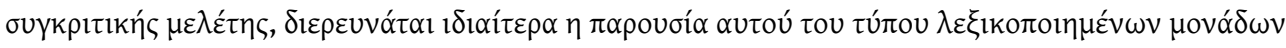

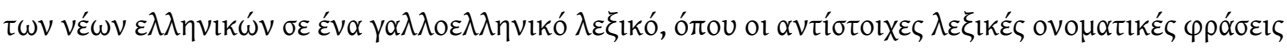

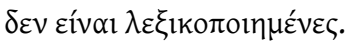

\section{INDEX}

Index géographique : Grèce

motsclesmk СЛОЖЕНИ ЗБОРОВИ, КУЛИНАРСТВО ЛЕКСИКОН, ДВОЈАЗИЧНИ ЛЕКСИКОГРАФИЈА, НОМИНАЛНА ФРАЗИ, ГРЦИЈА, ДВАЕСЕТТИОТ ВЕК, ДВАЕСЕТ И ПРВИОТ ВЕК, ЛИНГВИСТИКА

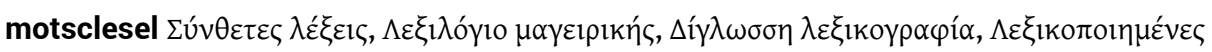

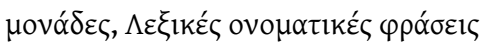

Keywords : Compounds, Culinary lexis, Bilingual lexicography, Lexicalized items, Nominal locutions

Mots-clés : mots composés, mots composés, lexique culinaire, lexique culinaire, lexicographie bilingue, lexicographie bilingue, unités lexicalisées, unités lexicalisées, locutions nominales, locutions nominales

Index chronologique : vingt-et-unième siècle motsclestr Bileşik sözcükler, Mutfak sözlüğü, İki dilli sözlükçülük, Nominal ifadeler, Yunanistan, Yirmi yüzyıl, Yirmi birinci yüzyıl, Dilbilim

Thèmes: Linguistique

\section{AUTEUR}

\section{GEORGES GALANÈS}

CREE, Inalco, USPC 